
\title{
25 Research Suare \\ 3D Culture and Characterization of Blood-Brain Barrier Endothelial Cells in a New Microfluidic Platform
}

\section{Sebastian Trennheuser}

Ruprecht Karls Universitat Heidelberg

\section{Felix Schmitt-Hoffner}

DKFZ Heidelberg

\section{Anne Mahringer}

Ruprecht-Karls-Universität Heidelberg

\section{Lena Mesch}

Natural and Medical Sciences Institute at the University of Tuebingen

\section{Peter Jones}

Natural and Medical Sciences Institute at the University of Tuebingen

\section{Martin Stelzle}

Natural and Medical Sciences Institute at the University of Tuebingen

Gert Fricker ( $\nabla$ gert.fricker@uni-hd.de )

Ruprecht Karls Universitat Heidelberg https://orcid.org/0000-0003-3894-961X

\section{Research}

Keywords: Blood-brain barrier, BBB, organ-on-a-chip, microfluidics, PBCEC, in vitro

Posted Date: November 3rd, 2020

DOl: https://doi.org/10.21203/rs.3.rs-100206/v1

License: (c) (i) This work is licensed under a Creative Commons Attribution 4.0 International License. Read Full License 


\section{Abstract}

Background: The blood-brain barrier (BBB) protects the central nervous system (CNS) from harmful substances in the peripheral blood stream and ensures brain homeostasis by regulating the transport of nutrients and metabolites. It also prevents many pharmaceutical drugs from reaching their target site and dysfunctions of the BBB are often associated with CNS diseases.

In order to provide a new in vitro platform that allows the investigation of barrier characteristics of the BBB and drug transport under physiological and pathological conditions, the TransBBB system was developed. The microfluidic chip consists of ten parallel culture chambers, each allowing the cultivation of endothelial cells on a physiological matrix under perfusion.

Methods: Porcine brain capillary endothelial cells (PBCECs) were cultivated on the surface of a hydrogel matrix resulting in a barrier which separates the culture chambers into two compartments. A protocol was established in order to obtain a tight cell layer under perfusion and the effect of different perfusion volumes on BBB formation was investigated. The barrier was then characterized in terms of cell viability, barrier integrity and expression of tight junction proteins.

Results: PBCECs formed a tight cell layer on the hydrogel surface preventing FITC dextran from permeating the cellular barrier. The presence of ZO-1 at the cell borders was shown indicating a functional BBB. The perfusion experiments suggested a critical role of medium volume for cell layer integrity under perfusion conditions.

Conclusions: The TransBBB chip presented here provides a promising, scalable microfluidic platform for the investigation of cellular barriers which can be integrated seamlessly into existing cell culture and drug testing workflows.

\section{Background}

The blood-brain barrier (BBB) is a highly selective cellular structure separating the central nervous system (CNS) from the peripheral blood stream. The so-called neurovascular unit (NVU) is composed of endothelial cells attached to a basal lamina, pericytes, parenchymal cells like astrocytes and extracellular matrix components $[1,2]$. Endothelial cells are connected by tight junction proteins like claudins or occludin preventing paracellular diffusion and resulting in high transendothelial electrical resistance (TEER) [3]. Furthermore, the endothelium expresses various efflux transporters like P-glycoprotein (P-gp), multidrug-resistance proteins (MRP) or breast-cancer-resistance proteins (BCRP) impeding transcellular permeation [4]. As a result, the blood-brain barrier upholds brain homeostasis and prevents possible harmful substances from reaching the brain, but also poses an obstacle for pharmaceutical agents intended for the treatment of CNS diseases [5]. Moreover, many diseases of the CNS are associated with dysfunctions of the BBB, e.g. Alzheimer's disease, Parkinson's disease etc. [6, 7]. 
Since many features of the BBB are difficult to assess in vivo, capable in vitro models are essential for the development of new drug candidates as well as the investigation of pathological conditions.

Within the scope of preclinical research, potential drug candidates are investigated in cell culture models and animals prior to their application to humans. Although animal models offer a complex organic environment, therapeutic effects as well as possible toxic side effects cannot be predicted sufficiently by animal experiments due to species related differences [8]. Furthermore, the ethical questions regarding the extensive use of animal trials have gained more and more attention in recent years.

The most common cell culture model for the investigation of physiological barriers is the Transwell system [9]. It is a well plate whose wells are divided into two chambers by a membrane. Endothelial cells are cultivated on the membrane allowing the investigation of transport processes across the cellular barrier. While it is easy to handle, suitable for high throughput and enables the possibility of using human cells, it lacks essential physiological features such as a physiological extracellular matrix or flow related effects like shear stress.

To overcome the shortcomings of traditional cell culture models like the Transwell system, several new microfluidic in vitro models, so-called organ-on-a-chip models, have been developed in recent years [1013]. The vision is the simulation of the smallest functional units of organs in order to investigate physiological and pathological conditions as well as drug transport mechanisms [14, 15]. An ideal organon-a-chip model should address the following requirements:

- standard format for seamless integration into existing workflows

- biocompatible, transparent material

- multiple culture chambers for high throughput assays

- integration of perfusion

- physiological extracellular matrix

- option for co-culture experiments

- real-time read-outs

- simple handling

Although remarkable progress has been made in this field in recent years, there is still a need for a system that meets all requirements previously mentioned. Many of the published microfluidic devices are made of polydimethylsiloxane (PDMS) due to its advantages for microfabrication, however, it is also known for the adsorption of hydrophobic compounds [16-20]. Other systems use artificial membranes for the cultivation of endothelial cells rather than a more physiological extracellular matrix [18-23]. Another disadvantage of some devices is the fact that they are connected to tubes and pumps which is complex to handle and therefore susceptible for disruptions.

In this study, a new microphysiological chip made of cyclic olefin polymer (COP) in microwell format produced by microinjection-molding was used to establish a blood-brain barrier model with brain capillary 
endothelial cells. One chip contains ten culture chambers and each culture chamber consists of two channels separated by an array of micropillars. One of the channels is filled with a biochemically functionalized hydrogel which forms an interface between two pillars. Endothelial cells are seeded on the hydrogel surface, thus forming a barrier between the two channels. The channel filled with hydrogel represents the abluminal and the other channel filled with medium the luminal side. The luminal channel can be perfused with cell culture medium by gravity driven flow induced by a difference of the medium amount in in- and outlet reservoir. By using a programmable rocker that tilts the chip at specific intervals at a certain angle, bidirectional perfusion can be controlled over long periods of time without the need of a complex periphery involving tubes and pumps.

A more comprehensive description of the technical details of the chip will be published elsewhere.

The aim of this study was to demonstrate the suitability and potential of the TransBBB system as a new in vitro model of the blood-brain barrier.

\section{Methods}

\section{Cell culture}

Primary porcine brain capillary endothelial cells (PBCECs) were isolated as previously described [24] and stored in liquid nitrogen. Brains were obtained by the slaughterhouse Mannheim free of charge. For every experiment freshly thawed PBCECs were used.

\section{Preparation of the Chips}

One channel of each culture chamber of the microfluidic TransBBB chip (microfluidic ChipShop GmbH, Jena, Germany) was filled with a mixture of the components of 3-D Life PVA-CD Hydrogel SG (Cellendes $\mathrm{GmbH}$, Reutlingen, Germany) and Matrigel (Corning, New York, USA). Matrigel was added to enhance cell adhesion. Since Matrigel becomes solid at temperatures above $8{ }^{\circ} \mathrm{C}$, all solutions and materials including the chip were pre-cooled overnight at $-20^{\circ} \mathrm{C}$. The additional movie file shows the gel filling procedure in detail (see Additional file 1). 30 minutes after gel filling the luminal channel was filled with cell culture medium (Earl's medium M199 supplemented with L-glutamin, Pen/Strep, Kanamycin and fetal bovine serum (FBS), Biochrom, Berlin, Germany). The chip was placed in the incubator $\left(37^{\circ} \mathrm{C}, 5 \% \mathrm{CO}_{2}\right)$ for $24 \mathrm{~h}$ to equilibrate the hydrogel in order to avoid the formation of air bubbles in the microfluidic channels. PBCECs in equilibrated medium were seeded at a density of $1,5 \times 10^{7}$ cells $/ \mathrm{mL}$. After a $5 \mathrm{~h}$ attachment period with the chip positioned on its short edge to allow the cells to sediment on the hydrogel surface, reservoirs were placed on in- and outlet. $60 \mu \mathrm{L}$ medium was filled in the inlet reservoir and $20 \mu \mathrm{L}$ extracted from the outlet reservoir in order to remove unattached cell debris. After $24 \mathrm{~h}$ of incubation, perfusion was started by placing the chip on a programmable rocker (Mimetas $\mathrm{GmbH}$, Leyden, Netherlands). It slowly tilted the chip $(0,1 \mathrm{cycle} / \mathrm{min})$ with $-18^{\circ}$ to $+18^{\circ}$ tilting angle resulting in gravity driven, bidirectional perfusion.

\section{Perfusion volume assay}


Preliminary experiments showed a high sensitivity of PBCECs when perfused with $1 \mathrm{~mL}$ of cell culture medium. Hence, the influence of the total volume of medium used for perfusion was investigated. Control channels were connected to $1 \mathrm{~mL}$ pipette tips as medium reservoir and filled with $1 \mathrm{~mL}$ medium. $10 \mu \mathrm{L}$ tips were used for low volume channels and filled with $20 \mu \mathrm{L}$ medium. Perfusion was induced by tilting the chip every $10 \mathrm{~min}$. The development of cell morphology was documented over a period of $30 \mathrm{~h}$. Medium was changed after $24 \mathrm{~h}$.

\section{Viability}

Calcein-Acetoxymethylester (calcein-AM) is a lipophilic, nonfluorescent compound which can be used to visualize viable cells. It is able to penetrate cell membranes where it is cleaved by unspecific intracellular esterases. The resulting calcein is hydrophilic and accumulates in the cytoplasm. In contrast to calceinAM, calcein is highly fluorescent and can be visualized with a fluorescence microscope.

Cells were washed by removing medium from the reservoirs, adding $60 \mu \mathrm{L}$ PBS to the inlet reservoir and waiting for PBS to pass the channel. After removing PBS from the reservoirs, cells were stained by adding Calcein-AM solution in medium $(4 \mu \mathrm{g} / \mathrm{mL})$ to the reservoir. After an incubation period of $30 \mathrm{~min}$ at $37^{\circ} \mathrm{C}$ cells were washed again and imaged with a Leica TCS SP5 II confocal microscope (CLSM).

\section{Barrier integrity - Permeability}

The integrity of cell layers can be assessed by perfusing the luminal channel with a fluorescent marker that cannot penetrate a confluent cell barrier and observing its permeation into the abluminal channel over time.

Cell culture medium was removed from the reservoirs and replaced with a solution of FITC dextran (20 kDa, 0,5 mg/mL, Sigma Aldrich, Germany). The permeation was documented by taking one picture per minute over 10 min with Leica CLSM. To quantify the permeation rate of FITC dextran into the hydrogel matrix, two regions of interest (ROI) were selected, one on the luminal side of a cell barrier and the other on the abluminal side. The fluorescence intensity of the luminal channel was related to the fluorescence intensity of the abluminal channel using the analyzing software FiJi. The relative fluorescence intensity was plotted over time using Microsoft Excel.

\section{Barrier characterization - Immunostaining of ZO-1}

The expression of various tight junction proteins is a pivotal characteristic of blood-brain barrier endothelial cells. Zonula occludens-1 (ZO-1) is an important tight junction protein connecting occludin and claudins to the cytoskeleton [25].

After carefully washing the cells with PBS, the reservoirs were removed. $10 \%$ neutral buffered formalin (NBF) was filled into the luminal channel and incubated for $10 \mathrm{~min}$ at room temperature to fix the cells. Blocking solution consisting of $1 \%$ goat serum and $0,25 \%$ TritonX in PBS was added and incubated for $1 \mathrm{~h}$ in order to permeabilize cell membranes and block unspecific binding sites. Primary anti-ZO-1 antibody with $0,5 \% \mathrm{BSA}$ and $0,125 \%$ TritonX was incubated for $24 \mathrm{~h}$ at $4{ }^{\circ} \mathrm{C}$ with the channels covered with parafilm to prevent evaporation of the antibody solution. Secondary antibody solution (goat, anti- 
mouse AlexaFluor 488) was incubated for $1 \mathrm{~h}$ at room temperature. Cells were washed after each incubation step with PBS and imaged with Leica TCS SP5 II confocal microscope.

\section{Results}

\section{Culture of PBCECs on TransBBB chip}

The result of the filling of the chip with hydrogel is shown in Fig. 2a. In order to improve visibility, the hydrogel was dyed with methylene blue. The hydrogel forms a smooth surface between pillars providing a culture area for brain endothelial cells. The seeding density of $1,5 \times 10^{7}$ cells $/ \mathrm{mL}$ is the highest possible density before clogging of the channels occurs. It was chosen in order to decrease the chance for uncovered hydrogel areas.

\section{Perfusion volume assay}

The morphology of cells perfused with $1 \mathrm{~mL}$ medium was compared to that of cells perfused with $20 \mu \mathrm{L}$. Since the hydrostatic pressure only depends on height (h), fluid density $(\rho)$ and gravitational acceleration $(\mathrm{g})$ but not on volume $(\mathrm{V})$, the perfusion rate was the same in control and low volume channels. As shown in Fig. 2c, cells perfused with $1 \mathrm{~mL}$ detached and perished shortly after starting perfusion. In contrast, cells perfused with $20 \mu \mathrm{L}$ remained attached and developed over time.

\section{Calcein-AM staining}

Calcein-AM was used to visualize viability and cell growth of PBCECs in the microfluidic channels of the TransBBB chip 4 days after seeding. As shown in Fig. 2b, cells grew on hydrogel surface as well as on pillars and channel walls. The side view was generated with CLSM by digitally assembling individual focal planes. It reveals that cells grew confluently on hydrogel surfaces while growth on pillars was less dense.

\section{Permeability}

FITC dextran $20 \mathrm{kDa}$ is a commonly used fluorescence marker that shows confluence of cell layers and makes any leaks visible. Figure $3 \mathrm{c}$ shows the permeation of FITC dextran into the hydrogel matrix in a control channel without cells, in a channel with a leaky barrier and across a tight barrier after $10 \mathrm{~min}$. Fluorescence intensity in the abluminal channel was considerably lower if a confluent cell layer grows on the hydrogel surface. In order to quantify the permeation of FITC dextran, the ratio of the fluorescence intensity in the abluminal channel and the luminal channel over time is shown in Fig. 3d. Channels with confluent cell layers showed significantly lower values than the cell free control channels indicating a tight barrier. Figure 3d also shows how leaky barriers lead to higher permeation rates.

\section{Immunostaining of ZO-1}

The expression of tight junction proteins is characteristic for brain capillary endothelial cells. The detection of tight junctions is therefore important for the evaluation of blood-brain barrier in vitro models. The result of the immunostaining of ZO-1 is shown in Fig. 3ab. A high fluorescence intensity can be seen on the hydrogel surface as the signals of several adjacent cells overlap. The detailed view of cells 
growing on the channel wall reveals the localization of the fluorescence signal. It shows that ZO-1 is located at the cellular borders as well as around the nuclei.

\section{Discussion}

The great demand for new in vitro models of the blood-brain barrier has led to the development of various interesting approaches and a remarkable progress in recent years. Microfluidic systems which are able to realistically simulate the smallest functional units of organs have the potential to revolutionize basic research as well as the development of new pharmaceutical drugs and carrier systems. This study introduces a new microfluidic in vitro model of the blood-brain barrier.

The TransBBB chip offers a platform with ten parallel culture chambers in microwell format which allows the seamless integration into existing cell culture workflows. Since it is made of COP, it can be produced by injection molding which makes it easy to manufacture on a large scale [26]. Compared to the frequently used PDMS, it also has the advantage of not exhibiting any adsorbing effects for hydrophobic substances [27]. This is particularly important considering the fact that most CNS drug are relatively hydrophobic [28]. It does not use an artificial membrane as culture area and instead relies on a hydrogel that mimics the physiological extracellular matrix. 3D Life biomimetic hydrogels from Cellendes ${ }^{\circledR}$ have been shown to successfully mimic extracellular matrices [29-31]. Including Matrigel as one of the gel components promotes cell adhesion and differentiation [32,33]. When filled into the chips with a small micropipette, it forms smooth surfaces between the pillars, offering physiological culture areas.

Formation of air bubbles poses a significant issue for microfluidic systems as they can impede perfusion and also impair cell growth [34]. It is important to know that the solubility of gases in liquids decreases with increasing temperatures. Therefore, cell culture medium used in this study was always equilibrated for at least $24 \mathrm{~h}$ in the incubator at $37^{\circ} \mathrm{C}$.

Perfusion is an important advantage of microfluidic systems compared to static models as it has been shown that the resulting shear stress on endothelial cells is a relevant factor for cell differentiation and the expression of tight junctions and transporters [35-37]. The TransBBB chip can be perfused by gravity driven flow induced by a programmable rocker without the use of complex tubing systems. This also allows perfusion with significantly lower medium volumes. The resulting shear stress on endothelial cells was calculated to be in a range from $0,05-0,1 \mathrm{~Pa}$, which is lower than shear rates found in vivo $(0,3-$ $2 \mathrm{~Pa})[37,38]$.

The comparison of cell morphology under perfusion with large and small volumes suggests the crucial role of the ratio of total volume to culture area. The relevance of the amount of cell culture medium used in microfluidic systems in general was already discussed by Walker et al. [34] and Sung et al. [39]. So far there is no conclusive explanation for the finding in our experiment but it will be subject of further investigations. However, the results of this experiment underline that it is an important aspect that should be taken into account when developing new microfluidic in vitro models. 
Cells with the greatest predictive value for blood-brain barrier models would be primary human brain capillary endothelial cells. Due to the difficulty of obtaining primary human cells, PBCECs were used for this study instead. It has already been shown that these have a greater relevance than immortalized cell lines in terms of barrier tightness and protein expression [40-43]. The field of induced pluripotent stem cells (iPSCs) has made a remarkable progress in recent years. iPSCs have the potential to show similar properties compared to primary cells while being readily available [44]. The use of iPSCs in the presented chip offers a promising option for the future.

PBCECs grew preferentially on the gel surface where they had a flat and stretched morphology leading to a dense layer that separated luminal and abluminal channel. The detection of ZO1 with an immunostaining demonstrates the formation of tight junctions. ZO-1 was also detected around the cell nuclei which could indicate a premature stage of cell development [45].

The permeation assay with the commonly used FITC dextran $20 \mathrm{kDa}$ as fluorescence marker enables the assessment of confluency and barrier integrity. The performed determination of the ratio of abluminal and luminal fluorescence intensity allows the evaluation of permeation rates [46].

Moreover, the TransBBB system offers integrated electrodes for in situ measurements of trans-endothelial electrical resistances (TEER). Up to now, no significant TEER values could be measured with PBCECs cultivated in the chip. It has been reported that even minimal gaps in cell layers could lead to drastically reduced TEER values [47]. Since one culture channel of the TransBBB chip contains up to 21 hydrogel surfaces, the probability is quite high that there could be a small gap in the cellular barrier on one of these surfaces. Therefore, the length of the individual channels and thus the number of cultivation areas per channel will be reduced in further developments. This will also allow even more culture chambers to be incorporated into one chip, which would enable greater throughput.

3-D Life biomimetic hydrogel contains peptide sequences which are cleavable by matrix-metalloproteases allowing cellular migration. The use of other cell types of the NVU like peri- or astrocytes is thus possible in order to further improve the relevance of the model.

\section{Conclusion}

This study presents a new approach of emulating the blood-brain barrier in vitro. This approach allows the cultivation of endothelial cells on a physiologically relevant matrix and perfusion without the need for a tubing periphery. It is built in microwell format and contains ten culture chambers allowing the integration in existing cell culture workflows and parallelization of experiments. It is planned to arrange the fluid ports in 96 well format which will further enhance the usability. The TransBBB system is suitable for the investigation of transport processes of new pharmaceutical drugs and carrier systems as well as the functionality of the BBB under pathological conditions like hypoxia or hyperglycemia and CNS diseases associated with dysfunctions of the BBB like Alzheimer's or Parkinson's disease. Apart from the blood-brain barrier, other relevant barriers such as gut- or kidney barrier could be emulated. 
The observation that PBCECs only survive when perfused with small medium volumes is a very interesting finding which will be further investigated in order to obtain a better understanding of the microenvironment in the microfluidic channels.

The discussed shortcomings will be addressed in future developments. Furthermore, the integration of other sensors for in situ monitoring of biorelevant parameters is planned.

All in all, the TransBBB provides a promising platform for the emulation and investigation of physiological barriers and it is a good starting point for further developments.

\section{List Of Abbreviations}

BBB: blood-brain barrier, CNS: central nervous system, NVU: neurovascular unit, TEER: transendothelial electrical resistance, P-gp: P-glycoprotein, MRP: multidrug resistance protein, BCRP: breast cancer resistance protein, PDMS: polydimethylsiloxane, COP: cyclic olefin polymer, PBCEC: porcine brain capillary endothelial cells, PVA: polyvinyl alcohol, SG: slow gelling, FBS: fetal bovine serum, Calcein-AM: calcein-acetoxymethylester, PBS: phosphate buffered saline, CLSM: confocal laser scanning microscopy, FITC: fluorescein isothiocyanate, ROI: region of interest, ZO-1: zonula occludens-1, NBS: neutral buffered formalin, iPSC: induced pluripotent stem cells

\section{Declarations}

\section{Ethics approval and consent to participate}

Not applicable

\section{Consent for publication}

Not applicable

\section{Availability of data and materials}

The datasets used and analysed during the current study are available from the corresponding author on reasonable request

\section{Competing interests}

The authors declare that they have no competing interests

\section{Funding}

This project was funded by Baden-Württemberg gGmbH (grant no. MIVT_002)

\section{Author's contributions}


Authors S.T., P.J., M.S. and G.F. designed the study. Authors S.T., F.S. and L.A. performed experiments and data analysis. Authors A.M., P.J., M.S., and G.F. supervised the research. Authors S.T., A.M., P.J., M.S. and G.F. wrote the paper.

\section{Acknowledgements}

The authors thank Mandy Lorenz for her help with the isolation of PBCECs and the Baden-Württemberg Stiftung for the financial support of this project within the research program "Mikrosystemtechnik für in vitro Testsysteme".

\section{References}

1. Sharif Y, Jumah F, Coplan L, Krosser A, Sharif K, Tubbs RS. Blood brain barrier: A review of its anatomy and physiology in health and disease: Blood Brain Barrier in Health and Disease. Clin Anat. 2018 Sep;31(6):812-23.

2. Hawkins BT, Davis TP. The Blood-Brain Barrier/Neurovascular Unit in Health and Disease. Pharmacol Rev. 2005 Jun;57(2):173-85.

3. Srinivasan B, Kolli AR, Esch MB, Abaci HE, Shuler ML, Hickman JJ. TEER Measurement Techniques for In Vitro Barrier Model Systems. J Lab Autom. 2015 Apr;20(2):107-26.

4. Mahringer A, Fricker G. ABC transporters at the blood-brain barrier. Expert Opin Drug Metab Toxicol. 2016 May 3;12(5):499-508.

5. Pardridge WM. Drug Transport across the Blood-Brain Barrier. J Cereb Blood Flow Metab. 2012 Nov;32(11):1959-72.

6. Neuwelt EA, Bauer B, Fahlke C, Fricker G, ladecola C, Janigro D, et al. Engaging neuroscience to advance translational research in brain barrier biology. Nat Rev Neurosci. 2011 Mar;12(3):169-82.

7. Sweeney MD, Zhao Z, Montagne A, Nelson AR, Zlokovic BV. Blood-Brain Barrier: From Physiology to Disease and Back. Physiol Rev. 2019 Jan 1;99(1):21-78.

8. Morris ME, Rodriguez-Cruz V, Felmlee MA. SLC, Transporters ABC. Expression, Localization, and Species Differences at the Blood-Brain and the Blood-Cerebrospinal Fluid Barriers. AAPS J. 2017 Sep;19(5):1317-31.

9. Audus KL, Borchardt RT. Characterization of an In Vitro Blood-Brain Barrier Model System for Studying Drug Transport and Metabolism. Pharm Res. 1986;03(2):81-7.

10. Modarres HP, Janmaleki M, Novin M, Saliba J, El-Hajj F, RezayatiCharan M, et al. In vitro models and systems for evaluating the dynamics of drug delivery to the healthy and diseased brain. J Control Release Off J Control Release Soc. 2018 Mar 10;273:108-30.

11. Bhalerao A, Sivandzade F, Archie SR, Chowdhury EA, Noorani B, Cucullo L. In vitro modeling of the neurovascular unit: advances in the field. Fluids Barriers CNS. 2020 Dec;17(1):22.

12. Bhatia SN, Ingber DE. Microfluidic organs-on-chips. Nat Biotechnol. 2014;32(8):760. 
13. Heidari H, Taylor H. Review Article: Capturing the physiological complexity of the brain's neurovascular unit in vitro. Biomicrofluidics. 2018 Sep;12(5):051502.

14. Esch MB, Smith AST, Prot J-M, Oleaga C, Hickman JJ, Shuler ML. How multi-organ microdevices can help foster drug development. Adv Drug Deliv Rev. 2014 Apr;69-70:158-69.

15. Huh D, Hamilton GA, Ingber DE. From 3D cell culture to organs-on-chips. Trends Cell Biol. 2011;21(12):745-54.

16. Toepke MW, Beebe DJ. PDMS absorption of small molecules and consequences in microfluidic applications. Lab Chip. 2006;6(12):1484.

17. Campisi M, Shin Y, Osaki T, Hajal C, Chiono V, Kamm RD. 3D self-organized microvascular model of the human blood-brain barrier with endothelial cells, pericytes and astrocytes. Biomaterials. 2018.

18. Griep LM, Wolbers F, De Wagenaar B, ter Braak PM, Weksler BB, Romero IA, et al. BBB on chip: microfluidic platform to mechanically and biochemically modulate blood-brain barrier function. Biomed Microdevices. 2013;15(1):145-50.

19. Jeong S, Kim S, Buonocore J, Park J, Welsh CJ, Li J, et al. A Three-Dimensional Arrayed Microfluidic Blood-Brain Barrier Model With Integrated Electrical Sensor Array. IEEE Trans Biomed Eng. 2018;65(2):431-9.

20. Sellgren KL, Hawkins BT, Grego S. An optically transparent membrane supports shear stress studies in a three-dimensional microfluidic neurovascular unit model. Biomicrofluidics. 2015 Nov;9(6):061102.

21. Walter FR, Valkai S, Kincses A, Petneházi A, Czeller T, Veszelka S, et al. A versatile lab-on-a-chip tool for modeling biological barriers. Sens Actuators B Chem. 2016;222:1209-19.

22. Brown JA, Pensabene V, Markov DA, Allwardt V, Neely MD, Shi M, et al. Recreating blood-brain barrier physiology and structure on chip: A novel neurovascular microfluidic bioreactor. Biomicrofluidics. 2015;9(5):054124.

23. Booth R, Kim H. Characterization of a microfluidic in vitro model of the blood-brain barrier ( $\mu \mathrm{BBB})$. Lab Chip. 2012;12(10):1784-92.

24. Mahringer A, Delzer J, Fricker G. A fluorescence-based in vitro assay for drug interactions with breast cancer resistance protein (BCRP, ABCG2). Eur J Pharm Biopharm. 2009 Aug;72(3):605-13.

25. Wolburg H, Lippoldt A. Tight junctions of the blood-brain barrier. Vascul Pharmacol. 2002 Jun;38(6):323-37.

26. Giboz J, Copponnex T, Mélé P. Microinjection molding of thermoplastic polymers: a review. J Micromechanics Microengineering. 2007 Jun 1;17(6):R96-109.

27. Niles WD, Coassin PJ. Cyclic Olefin Polymers: Innovative Materials for High-Density Multiwell Plates. ASSAY Drug Dev Technol. 2008 Aug;6(4):577-90.

28. Hansch C, Björkroth JP, Leo A. Hydrophobicity and Central Nervous System Agents: On the Principle of Minimal Hydrophobicity in Drug Design. J Pharm Sci. 1987 Sep;76(9):663-87. 
29. Rimann M, Graf-Hausner U. Synthetic 3D multicellular systems for drug development. Curr Opin Biotechnol. 2012 Oct;23(5):803-9.

30. Georgakopoulos N, Prior N, Angres B, Mastrogiovanni G, Cagan A, Harrison D, et al. Long-term expansion, genomic stability and in vivo safety of adult human pancreas organoids. BMC Dev Biol. 2020 Dec;20(1):4.

31. Shen S, Luo X, Gao K, Sun Y, Yao D, Zhu L. Identification and integrative analysis of microRNAs and mRNAs involved in proliferation and invasion of pressure-treated human liver cancer cell lines. Mol Med Rep [Internet]. 2019 May 9 [cited 2020 May 8]; Available from: http://www.spandidospublications.com/10.3892/mmr.2019.10223.

32. Hughes CS, Postovit LM, Lajoie GA. Matrigel. A complex protein mixture required for optimal growth of cell culture. PROTEOMICS. 2010 Feb 16;10(9):1886-90.

33. Kleinman HK, Martin GR. Matrigel: Basement membrane matrix with biological activity. Semin Cancer Biol. 2005 Oct;15(5):378-86.

34. Walker GM, Zeringue HC, Beebe DJ. Microenvironment design considerations for cellular scale studies. Lab Chip. 2004;4(2):91.

35. Cucullo L, Hossain M, Puvenna V, Marchi N, Janigro D. The role of shear stress in Blood-Brain Barrier endothelial physiology. BMC Neurosci. 2011 Dec;12(1):40.

36. DeStefano JG, Xu ZS, Williams AJ, Yimam N, Searson PC. Effect of shear stress on iPSC-derived human brain microvascular endothelial cells (dhBMECs). Fluids Barriers CNS. 2017;14(1):20.

37. Desai SY, Marroni M, Cucullo L, Krizanac-Bengez L, Mayberg MR, Hossain MT, et al. Mechanisms of Endothelial Survival Under Shear Stress. Endothelium. 2002 Jan;9(2):89-102.

38. van der Helm MW, van der Meer AD, Eijkel JC, van den Berg A, Segerink LI. Microfluidic organ-on-chip technology for blood-brain barrier research. Tissue Barriers. 2016;4(1):e1142493.

39. Sung JH, Wang Yl, Narasimhan Sriram N, Jackson M, Long C, Hickman JJ, et al. Recent Advances in Body-on-a-Chip Systems. Anal Chem. 2019 Jan 2;91(1):330-51.

40. Vastag M, Keseru GM. Current in vitro and in silico models of blood-brain barrier penetration: a practical view. Curr Opin Drug Discov Dev. 2009;12:115-24.

41. Aparicio-Blanco J, Martín-Sabroso C, Torres-Suárez A-I. In vitro screening of nanomedicines through the blood brain barrier: a critical review. Biomaterials. 2016;103:229-55.

42. Lundquist $\mathrm{S}$, Renftel $\mathrm{M}$. The use of in vitro cell culture models for mechanistic studies and as permeability screens for the blood-brain barrier in the pharmaceutical industry-Background and current status in the drug discovery process. Vascul Pharmacol. 2002 Jun;38(6):355-64.

43. Deli MA, Ábrahám CS, Kataoka Y, Niwa M. Permeability studies on in vitro blood-brain barrier models: physiology, pathology, and pharmacology. Cell Mol Neurobiol. 2005;25(1):59-127.

44. Appelt-Menzel A, Cubukova A, Günther K, Edenhofer F, Piontek J, Krause G, et al. Establishment of a human blood-brain barrier co-culture model mimicking the neurovascular unit using induced pluriand multipotent stem cells. Stem Cell Rep. 2017;8(4):894-906. 
45. Gottardi CJ, Arpin M, Fanning AS, Louvard D. The junction-associated protein, zonula occludens-1, localizes to the nucleus before the maturation and during the remodeling of cell-cell contacts. Proc Natl Acad Sci. 1996 Oct 1;93(20):10779-84.

46. Wevers NR, Kasi DG, Gray T, Wilschut KJ, Smith B, van Vught R, et al. A perfused human blood-brain barrier on-a-chip for high-throughput assessment of barrier function and antibody transport. Fluids Barriers CNS. 2018 Aug 31;15(1):23.

47. Odijk M, van der Meer AD, Levner D, Kim HJ, van der Helm MW, Segerink LI, et al. Measuring direct current trans-epithelial electrical resistance in organ-on-a-chip microsystems. Lab Chip. 2015;15(3):745-52.

\section{Figures}

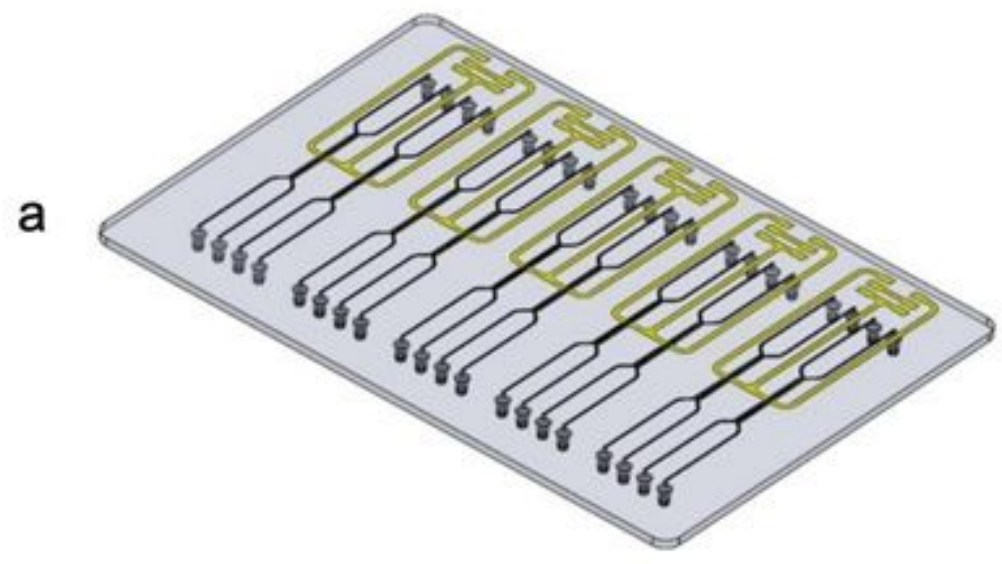

b

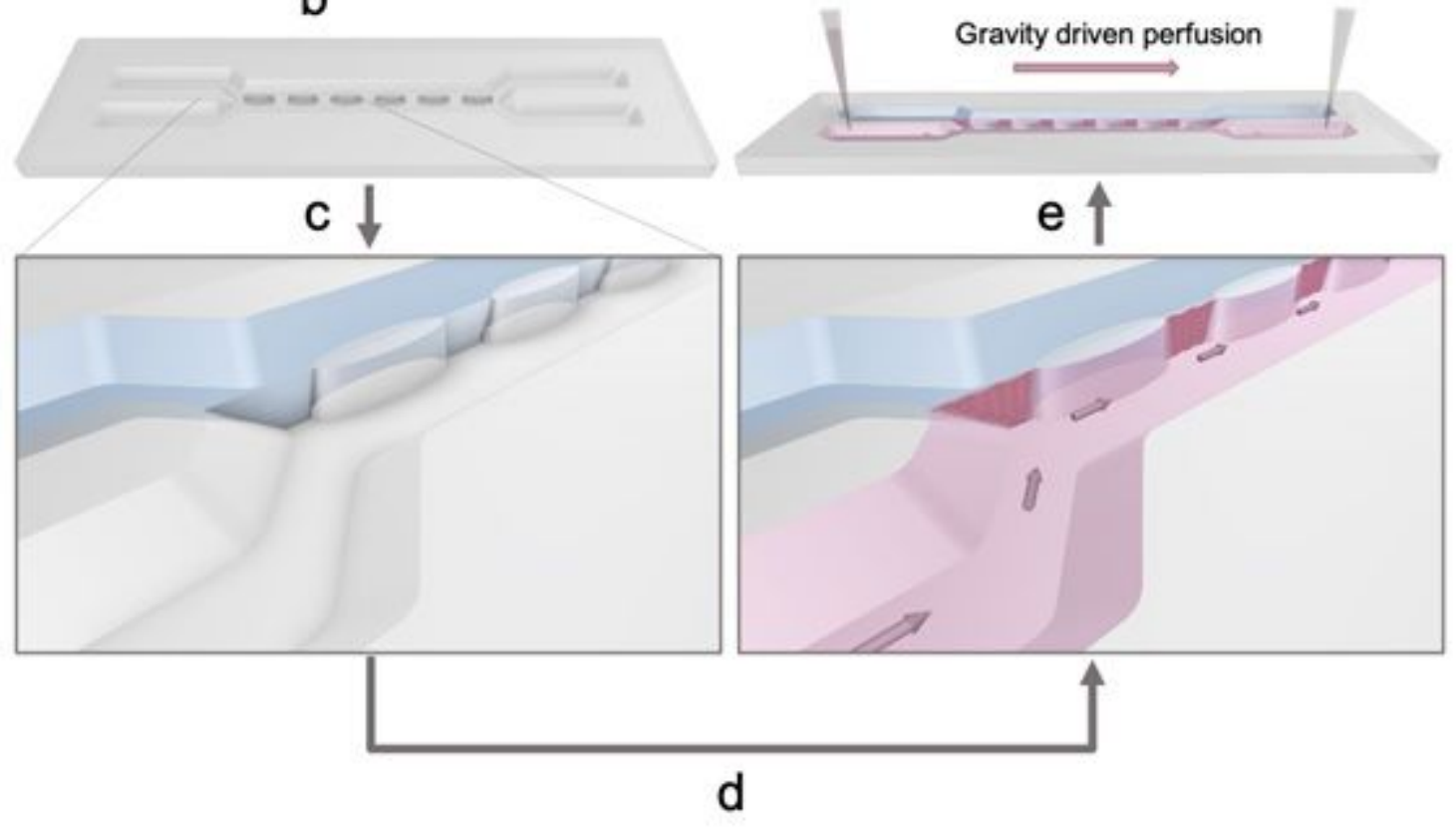

Figure 1 
TransBBB system a Microfluidic chip in microwell format with 10 parallel culture chambers and integrated electrodes (yellow) for TEER measurements. b Schematic illustration of one culture chamber. Two channels emerge and are then separated by a pillar array. c One channel is filled with a biomimetic hydrogel which forms an interface between the pillars due to surface tension. $d$ Endothelial cells are seeded on the hydrogel surface forming a barrier between the two channels. The hydrogel channel represents the abluminal side and the other channel the luminal side of the blood-brain barrier. e Different amounts of cell culture medium in in- and outlet reservoir induces gravity driven flow through the luminal channel simulating the blood stream.
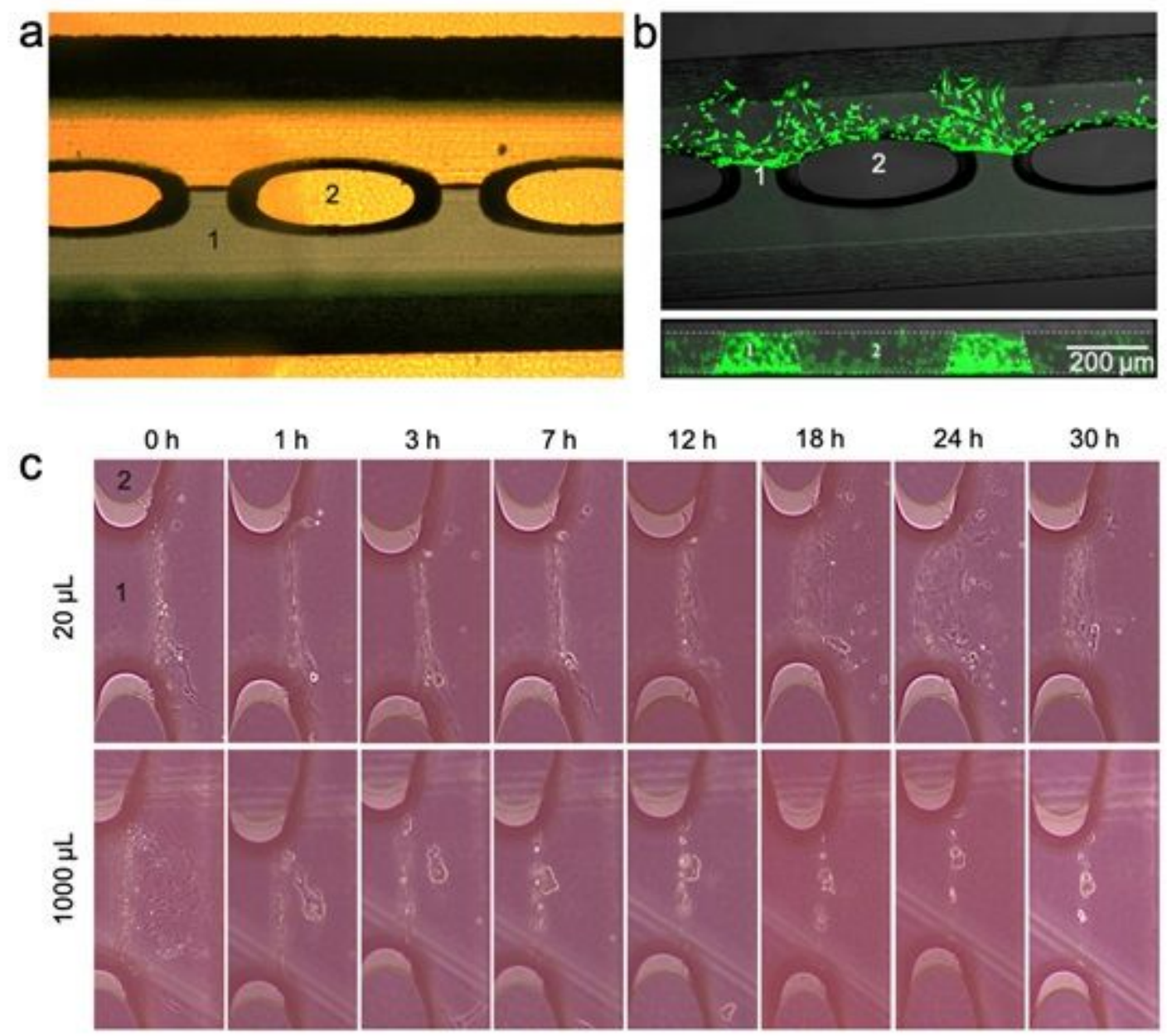

\section{Figure 2}

Filling of hydrogel and PBCEC morphology in microfluidic channels of TransBBB chips. a Hydrogel (1) was dyed with methylene blue for improved visibility. It forms smooth surfaces between the pillars (2). $b$ PBCECs were stained with calcein-AM to confirm viability and show cell growth in the microfluidic channels (top view and side view). c PBCECs were cultivated on hydrogel surfaces and perfused with different volumes of cell culture medium, $20 \mu \mathrm{L}$ and $1000 \mu \mathrm{L}$, at similar perfusion rates. Microscopic pictures were taken over a period of $30 \mathrm{~h}$ to document the development of cell morphology. 

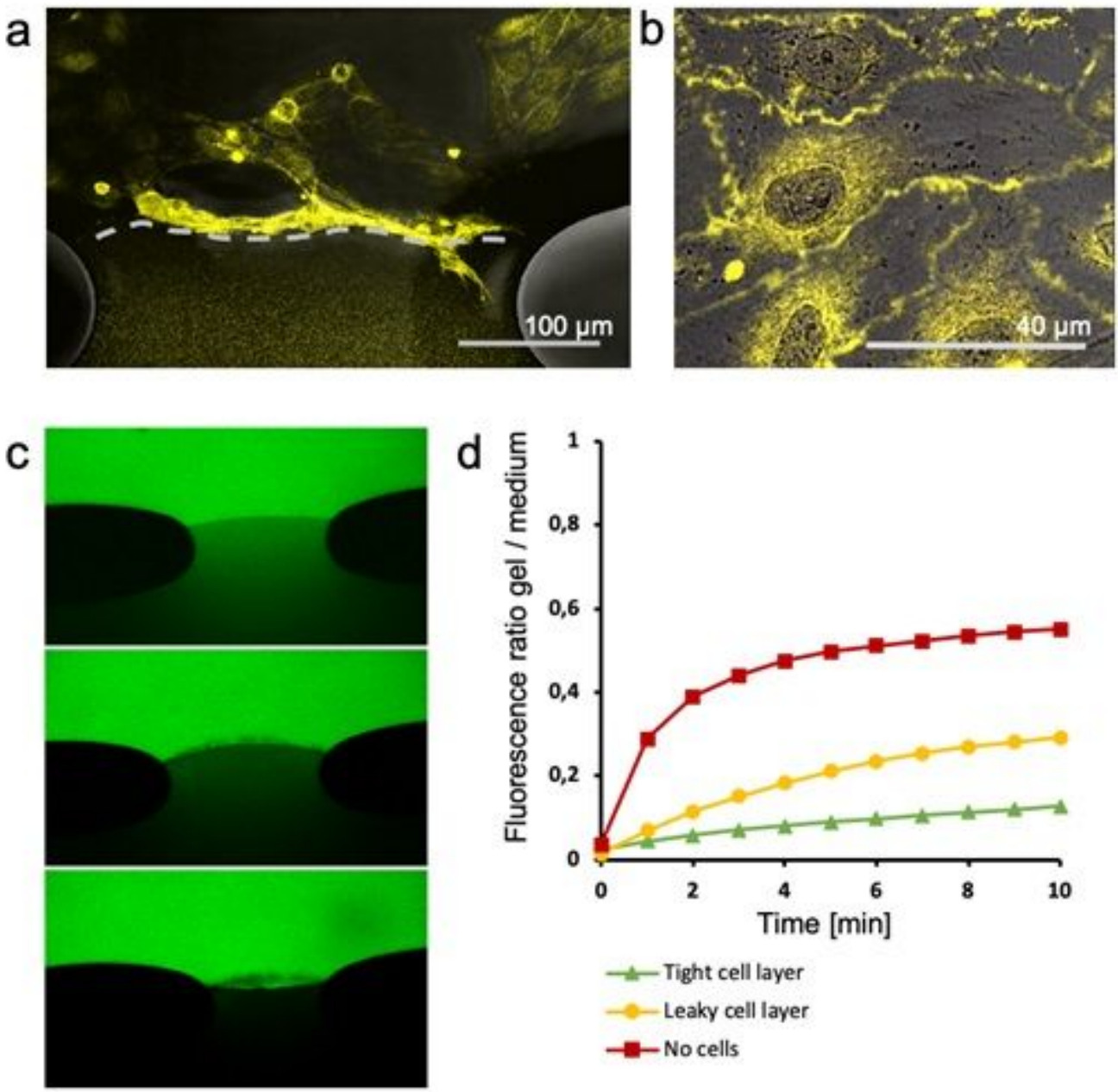

Figure 3

Characterization of PBCEC barrier in terms of tight junction expression and barrier integrity. a, b Overview and detailed view of the immunostaining of tight junction protein ZO-1. c Assessment of barrier integrity by perfusion with medium containing the permeation marker FITC dextran $20 \mathrm{kDa}$. Confluent cell layers prevent the permeation of FITC dextran into the hydrogel. Fluorescence microscopy pictures show a cell free control channel, a leaky barrier and a tight barrier. d Permeation of FITC dextran can be quantified by calculating the ratio of fluorescence intensity in the abluminal and the luminal channel. Permeation was observed over 10 min.

\section{Supplementary Files}

This is a list of supplementary files associated with this preprint. Click to download.

- Additionalfile1.m4v 